\title{
La Empresa Social Rural y su Contribución en la Conservación del Amaranto como Patrimonio Agroalimentario, Estudio de Caso: Centéotl, A.C. Zimatlán, Oaxaca, México
}

\section{The Rural Social Enterprise and its Contribution to the Conservation of Amaranth as an Agrifood Heritage, Case Study: Centéotl, A.C. Zimatlán, Oaxaca, Mexico}

\author{
Beatriz Rebeca Hernández-Hernández \\ beatrizrebecahernandez@gmail.com @ http://orcid.org/0000-0002-0804-7888 \\ Tecnológico Nacional de México, Instituto Tecnológico de Oaxaca \\ Calle Porfirio Díaz s/n. Santa María Vigallo, Zimatlán de Álvarez. CP 71200, México. \\ Juan Regino-Maldonado \\ jregino@ipn.mx @ https://orcid.org/0000-0003-2341-5703 \\ Instituto Politécnico Nacional, CIIDIR IPN UNIDAD OAXACA (Centro Interdisciplinario de Investigación \\ para el Desarrollo Integral Regional Unidad Oaxaca) \\ Calle Hornos 1003. Santa Cruz Xoxocotlán, CP 71230 Oax., México. \\ Andrés Enrique Miguel-Velasco \\ andres.miguel@itoaxaca.edu.mx @ http://orcid.org/0000-0003-1525-5017 \\ Tecnológico Nacional de México/Instituto Tecnológico de Oaxaca \\ Antequera 102, Col. Adolfo López Mateos, Oaxaca, Oax., CP 68030, México.
}

\section{INFO ARTÍCULO}

Enviado: 15/12/2019

Revisado: 14/02/2020

Aceptado: 15/02/2020

\section{PALABRAS CLAVE}

Economía Social y Solidaria

Empresa Social Rural

Desarrollo Rural

Seguridad Alimentaria

Amaranto (Amaranthus)

\section{KEYWORDS}

Social and Solidarity Economy

Rural Social Enterprise

Rural Development

Food Security

Amaranth (Amaranthus)

\begin{abstract}
RESUMEN
Este artículo describe las actividades que realiza la empresa social rural Centéotl, A. C. en Zimatlán, Oaxaca, México. Para promover la conservación del cultivo de amaranto como patrimonio agroalimentario. La metodología es cualitativa y descriptiva. El análisis por medio del paquete estadístico SPSS v.25 determinó la frecuencia de respuestas afirmativas con más del 50,0 por ciento, para precisar un impacto positivo. Los resultados mostraron que los bienes ofrecidos por la empresa contribuyen a la continuidad del cultivo (81,08 por ciento), y en menor medida servicios suministrados por Bancomunidad (51,3 por ciento). Este estudio de caso muestra que a través de técnicas de producción y consumo se puede adoptar este alimento en la dieta diaria. Se concluye que las actividades que realiza Centéotl promueven el reconocimiento de ese cultivo como patrimonio agroalimentario.
\end{abstract}

\begin{abstract}
This article describes the activities carried out by the rural social enterprise, Centéotl A. C. in Zimatlán, Oaxaca, Mexico. To promote the conservation of amaranth cultivation as an agrifood heritage. The methodology is qualitative and descriptive. The analysis by means of the statistical package SPSS v. 25 determined the frequency of affirmative answers with more than 50 percent, to specify a positive impact. The results showed that goods offered by the company contribute to the continuity of the crop ( 81,08 percent), and to a lesser extent services offered by Bancomunidad $(51,3$ percent). This case study shows that through production and consumption techniques this food can be adopted in the daily diet. It is concluded that the activities carried out by Centéotl promote the recognition of this crop as an agrifood heritage.
\end{abstract}




\section{INTRODUCCIÓN}

En México la revolución verde (a partir de 1943) construyó un tipo de desarrollo rural centrado en la tecnología y en el incremento de los rendimientos de la producción agrícola. Tradición que hasta nuestros días supone un impacto positivo en el desarrollo económico de los países en vías de desarrollo, sin importar los efectos nocivos en el medio ambiente y la sanidad alimentaria (Herrera, 2012).

Las nuevas técnicas de producción agrícola se fueron incorporando con posterioridad a otros países del tercer mundo con un aumento significativo en la producción de alimentos. Tal parece que la producción y distribución masiva de los mismos, continúan siendo los principales mecanismos de combate al hambre y pobreza no únicamente en los países más desfavorecidos sino en el mundo (Herrera, 2012). Sin embargo, las hambrunas continúan siendo un gran problema a resolver no sólo en algunos países de América Latina, sino también en países de África y Asia; aun cuando en la actualidad la tecnología y avances en ingeniería genética son notables, paradójicamente no se han tenido los avances esperados en materia de cobertura alimentaria (Todaro y Smith, 2015; Banerjee et al., 2006).

Un estudio realizado por Shamah et al. (2015) muestra que, en el ámbito internacional, 178 millones de niños menores de cinco años padecían desnutrición crónica (baja talla para la edad), responsable aproximadamente de 3,5 millones de muertes. Sostiene que, en México cerca de 15 millones de niños padecen desnutrición y es prevaleciente en estados de la región sur del país en zonas con gran población indígena. En 2015 de acuerdo con la Secretaría de Bienestar (anteriormente Secretaría de Desarrollo Social), de los 570 municipios con los que cuenta el estado de Oaxaca, 291 (51 por ciento) fueron atendidos por el Programa Cruzada Nacional contra el hambre, de los cuales, alrededor del 10 por ciento de los municipios fueron considerados prioritarios por presentar pobreza extrema alimentaria (SEDESOL, 2016).

El abandono del campo por numerosos agricultores en situación de desempleo, la debilidad demográfica y el envejecimiento de la población rural han definido un espacio marginal y fragmentado en las comunidades; así como los factores de cambio climático han agudizado el problema de seguridad alimentaria principalmente en los países pobres (Comité de Seguridad Alimentaria Mundial, 2012).

Debido a lo anterior, la planificación de las economías rurales y propuestas de sistemas de producción alternos al sistema económico convencional cobran relevancia en el desarrollo local con una visión de adentro hacia afuera, con la posibilidad de crear nuevos escenarios de desarrollo inclusivo que integren lo rural en lo global (Márquez, 2002).

Una respuesta al problema anterior se plantea desde la economía solidaria, situada como el conjunto de prácticas autogestionadoras, solidarias, democráticas y humanistas, sin ánimo de lucro en el desarrollo integral del ser humano. Esta corriente de la economía busca la producción, comercialización, consumo y la vida en sociedad a través de emplear métodos de producción optimizadores de insumos, y bajos en emisiones de carbono, sustentada en los principios de respeto, cuidado y conservación de la biodiversidad (Salcedo y Marcillo, 2010; Askunze, 2007).

Una propuesta desde esta perspectiva teórica, alterna al sistema de producción y abastecimiento tradicional es la creación de sistemas de redes alimentarias asociadas al comercio justo, la compra solidaria y al aprovisionamiento colectivo; mismas que ponen de manifiesto el papel de la solidaridad en la promoción de sistemas agroalimentarios locales (SIAs). Cuyo objetivo es la producción sustentable de alimentos básicos nutritivos procedentes particularmente de áreas rurales como frutos, granos, hortalizas, bebidas, carne entre otros, que enmarcados en un sistema sociocultural y ambiental son transformados en comida para autoconsumo, donde el excedente se destina al mercado (ONU, 2014).

En los SIAs el papel principal lo tienen los productores, quienes se organizan en las conocidas como empresas sociales rurales (ESR), en torno a bienes de propiedad privada y algunas de propiedad común; llamadas también laboratorios socioambientales fundamentadas en los tres ejes de sustentabilidad de la economía solidaria: social, ambiental y económico (Toledo, 2012).

Uno de los objetivos principales de las ESR es la producción para el autoconsumo. Productos que mejoren la alimentación y disminuyan la desnutrición en los niños, además de contribuir a la seguridad alimentaria de los pobladores (Martínez, 2016; Shamah et al., 2015; Toledo, 2012). 
Toledo (2012) propone el rescate los sistemas de producción agrícola tradicionales. Algunos estudios han reportado que el amaranto un cultivo ancestral de los pueblos indígenas de Mesoamérica estaba incluido en la dieta diaria de los pobladores por los beneficios a su alimentación, nutrición y salud (Martínez, 2016).

Así pues, el presente estudio destaca el papel que desempeñan las ESRs en la continuidad del cultivo de amaranto en las comunidades rurales por cuatro razones fundamentales: i) constituye una opción de solución al problema de seguridad alimentaria, principalmente en aquellas localidades en condiciones de vulnerabilidad, agravado por el fenómeno de cambio climático; ii) disminuye la desnutrición principalmente en niños y demás población vulnerable; iii) se protegen, conservan y valoran alimentos, y preparaciones domesticas derivadas de la agricultura familiar, presentes en varias regiones de países con ingresos bajos, de gran riqueza cultural y gastronómica. De acuerdo con Montecino (2015), un producto alimenticio será considerado patrimonio por una comunidad cuando éste se inserta de manera temporal, y adquiere valor simbólico como marca de identidad de la comunidad, que es quien lo produce, consume y comercializa. En consecuencia, contribuye al rescate del patrimonio agroalimentario regional para la seguridad alimentaria; y iv) la comercialización del grano mejora las condiciones económicas de las zonas productoras. Genera empleos que permiten anclar a las personas a sus lugares de origen y disminuir la migración de la población rural a las grandes ciudades (Martínez, 2016).

El trabajo se divide en tres apartados, en el primero se revisa la literatura sobre la empresa social rural y el patrimonio agroalimentario. En el segundo se detalla la metodología cualitativa utilizada en la investigación. Finalmente, en el tercero se presentan los resultados obtenidos, discusión y conclusiones del caso de estudio.

\section{REVISIÓN DE LITERATURA}

\subsection{Empresa social rural}

Las empresas sociales rurales (ESRs) son unidades económicas organizadas en torno a bienes de propiedad privada y común, en formas colectivas de producción que buscan generar bienes y servicios bajo los principios de sustentabilidad. En las que utilizan técnicas agroecológicas de producción para el mercado orgánico y justo (Herrera, 2012).

En México las encontramos en forma de cooperativas, comunidades o asociaciones diversas dedicadas a la producción orgánica de café, cacao, miel, y otros productos agrícolas. También dedicadas a la conservación comunitaria como el manejo de selvas y bosques, ecoturismo, pesca responsable y al manejo de fauna silvestre (Ortiz, 2015). A nivel nacional las ESRs se concentran principalmente en las regiones del centro y sur del país, ubicadas estratégicamente por su valiosa contribución al desarrollo local y regional. En 2016 el país contaba con 670 ESRs activas de un total nacional de 1180 unidades económicas (Tabla 1).

Tabla 1. Empresas sociales rurales en México 2014-2016.

\begin{tabular}{|c|c|c|c|c|c|}
\hline \multicolumn{2}{|c|}{ Nacional } & \multicolumn{2}{c|}{ Estatal } & \multicolumn{2}{c|}{ Regional } \\
\hline Total & Activas & Total & Activas & Total & Activas \\
\hline 1180 & 670 & 201 & 112 & 181 & 92 \\
\hline
\end{tabular}

Fuente: Elaboración propia, datos obtenidos Ibero-Puebla.

Para lograr el desarrollo rural y garantizar la seguridad alimentaria en las economías subdesarrolladas es necesario el empoderamiento de los trabajadores y pequeños agricultores (ONU, 2014). Cobra importancia la construcción de redes alimentarias alternativas de producción sostenible, integrada por ESRs con prácticas de comercio justo, compra solidaria y abastecimiento colectivo donde se pone de manifiesto el papel de la solidaridad en la promoción de sistemas agroalimentarios más justos. 


\subsection{Patrimonio agroalimentario}

El patrimonio cultural se concibe como aquello que socialmente se considera digno de conservación independientemente de su interés utilitario (Llorenc, 1998). El estilo e identidad culinaria son elementos de las culturas de familias, localidades y regiones, desde allí se gestan, conservan y expanden o permanecen como particularidades de un lugar o de un territorio, pueden ser considerados como valores intangibles de las comunidades rurales. Un alimento para el ser humano será considerado patrimonio por una comunidad, cuando ésta lo adopte en su dieta de manera continua por su valor nutricional, simbólico, de creación y fortalecimiento de la identidad cultural de sus pobladores (Echeverría, 2015).

El patrimonio agroalimentario se da cuando la producción de alimentos y el procedimiento para ser transformados en comida se gesta dentro de un sistema cultural, que les otorga significados asentados en una historia en particular de cambios, continuidades tecnológicas y simbólicas. Contribuye a la revalorización continua de las entidades y culturas de los pueblos, se constituye como el capital cultural de las sociedades contemporáneas y por lo tanto, también es fuente de inspiración para la creatividad y la innovación de productos culturales contemporáneos y futuros (UNESCO, 2018).

\subsubsection{El amaranto patrimonio cultural inmaterial}

El patrimonio cultural inmaterial proporciona a los pobladores de las comunidades identidad a través de la conservación, preservación y expansión de numerosos saberes tradicionales o autóctonos que, favorecen a la creatividad, bienestar social y contribuyen a la gestión del entorno natural y social, incluidas políticas sanitarias, de educación y gestión de recursos naturales. Además de generar empleos e ingresos económicos para los más pobres y vulnerables (UNESCO, 2015).

Hablar de patrimonio cultural inmaterial es plasmar la tradición y la memoria de la actividad artesanal de producción del amaranto, que se ha transferido de generación en generación de padres a hijos y representa el sustento de muchas familias (UNESCO, 2017).

El amaranto es un cultivo ancestral, planta del género amaranthus llamada comúnmente alegría, quelite, quintonil o bledo (imagen 1).

En 2016 por primera vez se muestran alrededor de 50 fósiles de semillas prehispánicas que evidencia el origen de la agricultura en el país (maíz, calabaza, aguacate, chuspata, algodón y amaranto), que datan aproximadamente desde hace nueve mil años a. C., encontradas en las cuevas de Coxcatlán en Tehuacán (Puebla) y de Guila Naquitz (Oaxaca) (INAH, 2016). En ese mismo año el amaranto fue reconocido como patrimonio cultural intangible de la Ciudad de México. Se reconoció su importancia cultural, a la memoria histórica que está detrás del cultivo, y fundamentalmente a la conservación de saberes y conocimientos para trasformar el amaranto en un producto de consumo final, de esta manera constituirse como patrimonio agroalimentario de la humanidad (Hernández et al., 2019).

Desde la época prehispánica y hasta nuestros días, la semilla de amaranto ha sido uno de los alimentos más importantes en la dieta de las culturas mesoamericas. En México es importante en la agricultura tradicional, resurge en los últimos años gracias al esfuerzo y solidaridad de mujeres interesadas en regresar a sus raíces ancestrales con el cultivo y consumo de alimentos sanos. La producción de amaranto, los conocimientos, las habilidades y las prácticas autóctonas, mantenidas y mejoradas a lo largo de diferentes generaciones han proporcionado medios de subsistencia para sus familias.

\subsection{Empresa social rural y patrimonio agroalimentario}

La economía solidaria plantea que la creación y sostenimiento de redes de sistemas agroalimentarias locales (SIAL) integradas por empresas sociales rurales bajo los principios de la sustentabilidad pueden conducir a lograr el desarrollo rural, y enfrentar los retos de la economía abierta, los desafíos del cambio climático y de seguridad alimentaria en las economías de países en vías de desarrollo (Toledo, 2012). 
Un SIAL es un tejido empresarial localizado en un territorio donde existe especialización y concentración local relativamente alta con una determinada producción agroalimentaria. Constituido por aprovechamientos agrarios, cooperativas agropecuarias, asociaciones de productores orgánicos, pequeñas y medianas empresas agropecuarias, agroindustrias, comercializadoras, prestadoras servicios de ecoturismo y de microfinanzas; todas las anteriores unidades económicas conocidas también como empresas sociales rurales que muestran un nivel alto de interrelación y de colaboración entre sí (Sanz, 2010).

EI SIAL mantiene vínculos muy estrechos con instituciones locales, públicas, agencias descentralizadas de gobierno, organizaciones no gubernamentales, instituciones de, fomento de la tipicidad y calidad alimentaria, grupos y asociaciones de desarrollo rural, instituciones de formación de recursos humanos, transferencia y difusión de tecnologías adecuadas e innovaciones. Las instituciones y agentes anteriores pueden construir sinergias colectivas, cristalizadas en acciones y proyectos comunes de desarrollo agroalimentario y rural.

En las zonas rurales donde hay concentración local y cierto grado de especialización agroalimen-

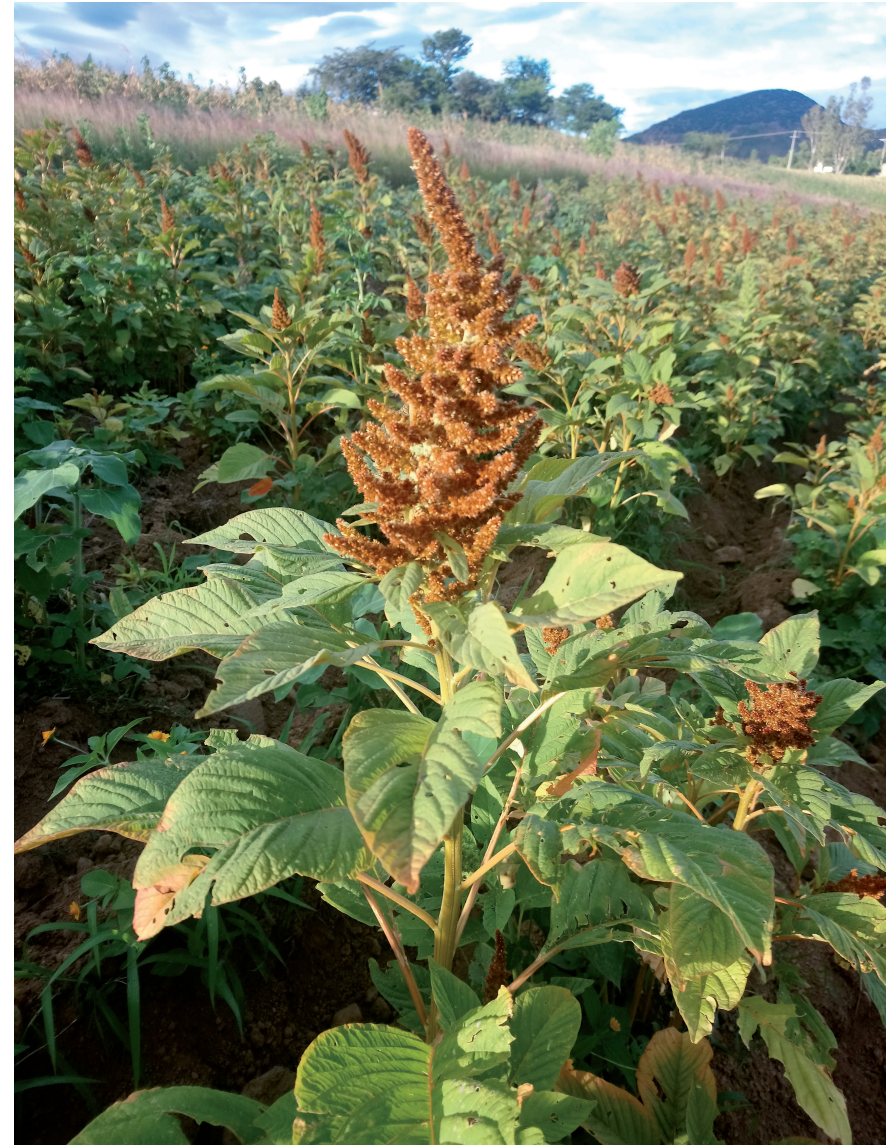

Imagen 1. Planta de amaranto, variedad dorado. Fuente: Autor. taria, se pueden tener mayores posibilidades de obtener ventajas competitivas, gracias a las relaciones de proximidad, mediante estrategias de producción y calidad vinculadas al territorio, a diferencia de otros ámbitos geográficos. Las ESRs luchan ante la visión extensionista de la industria alimentaria y poder de la publicidad para comida procesada y chatarra. Las estrategias competitivas basadas en la diferenciación sociocultural de alimentos apoyarían a desvincular este tipo de demanda (comida procesada), a una cuyo factor de diferenciación sea de carácter sociocultural y territorial. Donde se prefieran consumir alimentos diferenciados culturalmente, se identifiquen las prácticas tradicionales de producción y conservación de alimentos en los lugares de origen, como un indicador confiable de calidad y de seguridad de los productos (Sanz, 2002).

Algunas empresas han desarrollado estrategias para preservar y proteger el patrimonio agroalimentario, protegiendo sus productos de imitaciones y falsificaciones, alegrando el paladar de los consumidores mediante signos distintivos de sabor y calidad, denominación de origen, indicaciones geográficas protegidas, marcas territoriales, entre otras (Sanz, 2010).

De igual forman con el objetivo de proteger el patrimonio agroalimentario de algunas comunidades, estratégicamente otras empresas han diversificado los productos derivados del amaranto, aprovechado su valor nutricional. Diversos estudios han evidenciado que los valores nutricionales del amaranto, lo sitúan como uno de los alimentos con alto potencial agroalimentario, debido a que, la semilla, planta y hojas poseen valores nutricionales que sobrepasan a algunos cereales de consumo común. Derivado de ello, es ampliamente recomendado para consumo humano, además de ser una semilla que no contiene gluten en su composición química, adecuado para las personas que presentan intolerancia a este componente (De Jesús et al., 2017; Martínez, 2016).

En Colombia la ESR Agrosolidaria rescata el conocimiento tradicional con la conservación de ecosistemas ambientales para fomentar la producción, consumo y comercio de alimentos nativos (CAC, 2015). En este mismo país, Nutri q life a través de la conservación de reservas ambientales productivas de amaranto, cuya 
misión es cultivar, procesar y comercializar productos alimenticios derivados del amaranto, se mejora la calidad de vida de la población y conserva el patrimonio agroalimentario (Molano, 2018). En Salta, Argentina, se fomentan los emprendimientos sociales comunitarios de agricultura autóctona como la producción de kiwicha o amaranto, en comunidades marginadas para conservar su patrimonio agroalimentario y mejorar su bienestar (Paredes y Sato, 2018).

En México desde la década de los ochentas, la sociedad civil y el sector privado han impulsado el cultivo de amaranto, su difusión y comercialización, aunque no de manera sostenida; un ejemplo es la ESR Centéotl localizada en Oaxaca quien ha logrado la producción colectiva y diversificación de productos derivados del amaranto. En el país, como respuesta a la problemática alimentaria, la política de producción agrícola está revalorando aquellos alimentos producidos por el pequeño productor que han ido perdiendo importancia en el consumo de las personas pero que, forman la identidad de las comunidades por su carácter tradicional y local. La estrategia impulsa la creación de cadenas cortas agroalimentarias de comercio justo y solidario para lograr el éxito en el mediano y largo plazo, fortaleciendo los lazos de comunicación, de intercambio y compromiso entre productores y consumidores. Cadenas de producción y comercialización de alimentos con métodos de producción agroecológicos, mejora de la calidad de los alimentos, generación de mercados de precio justo, y disminución o eliminación de intermediarios (Hernández et al., 2019).

En Chile, desde el año 2006, la Fundación para la Innovación Agraria, y el Programa Institucional de Innovación Estratégica de Patrimonio Alimentario han elaborado una serie de inventarios a nivel regional para promover la protección, conservación y valor del acervo cultural de los productos y alimentos distintivos de sus territorios (Pallacán, 2016).

De acuerdo con los anteriores estudios empíricos en América Latina, desde la sociedad civil las empresas sociales localizadas en áreas rurales, y a nivel nacional aperando en redes de sistemas agroalimentarias contribuyen en la revalorización de alimentos nativos nutritivos como el amaranto, con ello garantizan la seguridad alimentaria, y la conservación del patrimonio agroalimentario de las comunidades (Paredes y Sato 2018; Molano, 2018; Pallacán, 2016; Montecino, 2015; CAC, 2015).

De acuerdo con lo anterior, el presente documento plantea como hipótesis central que la empresa social rural Centéotl en Oaxaca (México), realiza actividades que promueven la conservación del cultivo de amaranto, contribuye a la seguridad alimentaria y al patrimonio agroalimentario de la humanidad (Figura 1).

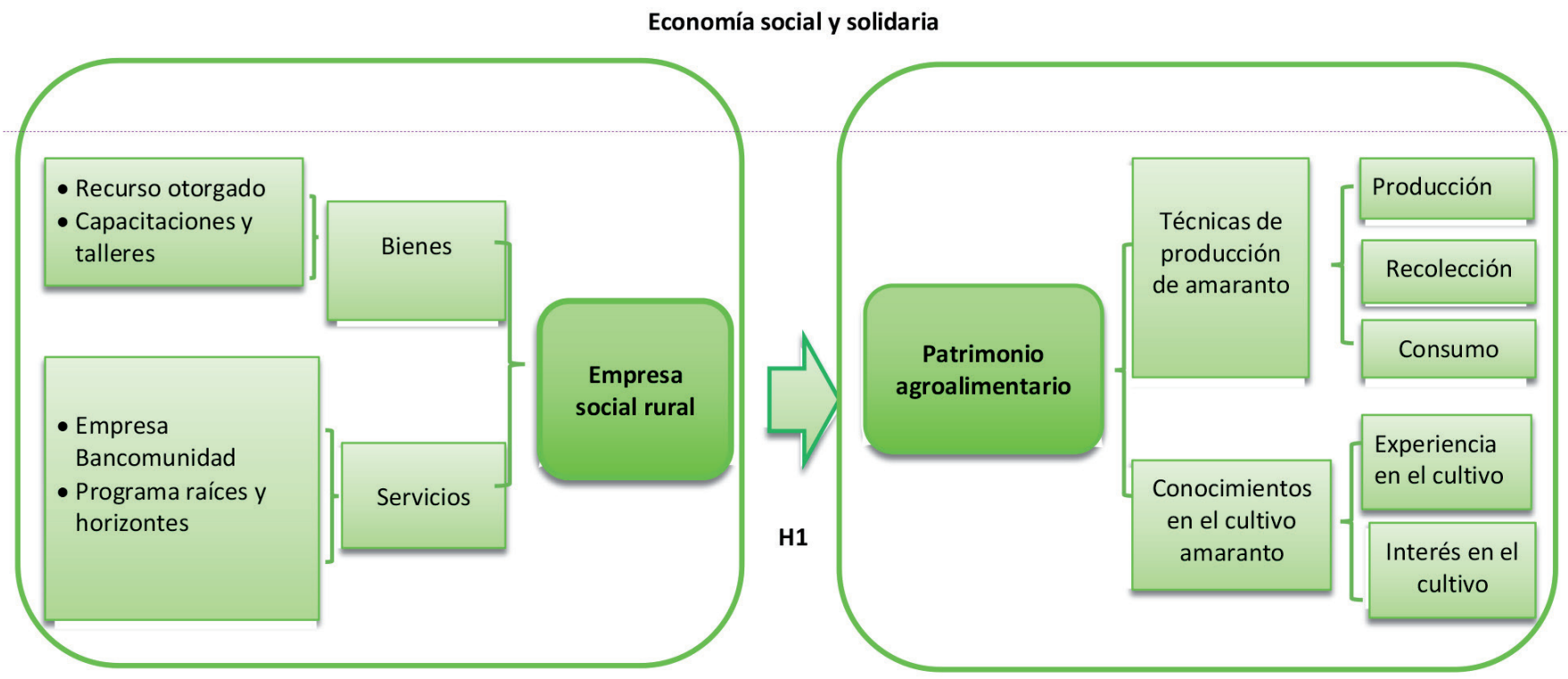

Figura 1.Modelo teórico metodológico. Centéotl A.C. Y la conservación del patrimonio agroalimentario. Fuente: Elaboración propia de acuerdo con los autores Echeverría et al., 2015; Toledo, 2012. 


\section{METODOLOGÍA}

El tipo de investigación fue cualitativa, la cual de acuerdo a Vasilanchis (2006) es multimetódica, naturalista e interpretativa, abarca el estudio, uso y recolección de una variedad de materiales empíricos que permiten mayor acercamiento al objeto de estudio. Se utiliza el estudio de caso, estrategia que consiste en abordar fenómenos contemporáneos de forma empírica en términos holísticos y significativos (Yin, 1994).

El estudio de caso se llevó a cabo en la empresa social rural Centéotl en Zimatlán, Oaxaca, con la intención de describir y explicar las variables a investigar. Lo anterior, implicó la recolección y análisis de datos cualitativos (Hernández, 2010; Yin, 1994). Para conocer las actividades que realizan los productores de amaranto que suministran el grano a la empresa social rural se utilizaron dos técnicas de recolección de información. La observación participante, es decir, aquella que permitió la obtención de información y producción de datos por tratarse de un proceso reflexivo entre los sujetos estudiados y el sujeto cognoscente (Guber, 1991).

Derivado de ello, se plantea el siguiente supuesto H1. La empresa social rural Centéotl A.C realiza actividades que promueven la conservación del cultivo de amaranto como patrimonio agroalimentario.

La entrevista semiestructurada se aplicó en el periodo de enero-agosto 2019 a través del análisis de la percepción de productores con la finalidad de conocer el vínculo de la empresa social rural con la conservación del patrimonio agroalimentario en 37 familias productoras de amaranto que trabajan con Centéotl. Esta constó de 13 preguntas, las 4 primeras permitieron investigar sobre las variables de empresa social rural presente y programas que lleva acabo Centéotl para asegurar la alimentación de la población en las comunidades, y de qué manera contribuyen a la conservación del cultivo. En las siguientes 9 preguntas se analizan las variables de patrimonio agroalimentario.

Para el análisis de la información las preguntas y sus respuestas, se concentraron en una base de datos en el paquete estadístico IBM (SPSS) v.25 y Microsoft Excel 2013, donde se analizó cada pregunta con las respuestas de los productores entrevistados, teniendo como guía el modelo teórico metodológico. Con la base de datos se determinó la frecuencia de acuerdo a la percepción del productor, donde si las respuestas contribuían afirmativamente con más del 50 por ciento, éstas ayudaban a precisar que la empresa Centéotl A.C. debe de considerarse como una ERS con un impacto positivo en la conservación del cultivo de amaranto como patrimonio agroalimentario.

\section{RESULTADOS}

Centéotl se fundó en el año 1994 con el objetivo de mejorar la calidad de vida de las comunidades, a través de implementar actividades en temas como seguridad alimentaria, equidad de género, democracia y protección al medio ambiente. Esta empresa se ubica en Zimatlán de Álvarez, Oaxaca, México (imagen 2).

A continuación, se describen las variables analizadas en este documento, que fueron estructuradas a partir de un procedimiento analítico, descriptivo y documental con la finalidad de dar respuesta a la hipótesis anterior $\mathrm{H} 1$.

La empresa promueve la revalorización del cultivo de amaranto, por medio de bienes y servicios, a través de recursos, talleres y capacitaciones relacionados con el cultivo de amaranto. Así mismo, por medio de la empresa Bancomunidad ofrece el servicio de microcréditos con tasas de interés bajas, mismos que pueden ser adquiridos por un grupo de 5 mujeres que, en primer lugar, deseen ser socias y, segundo lugar, existan lazos de amistad y confianza, lo que a su vez permitirá el compromiso de pago del crédito. Finalmente, por medio del programa Raíces y Horizontes, impulsa la integración de actividades productivas, culturales y cuidado del medio ambiente, así como la producción y consumo de amaranto en niños, jóvenes y adultos para mejorar su calidad de vida y arraigarlos a su comunidad. 
Imagen 2. Localizacion Centéotl A.C

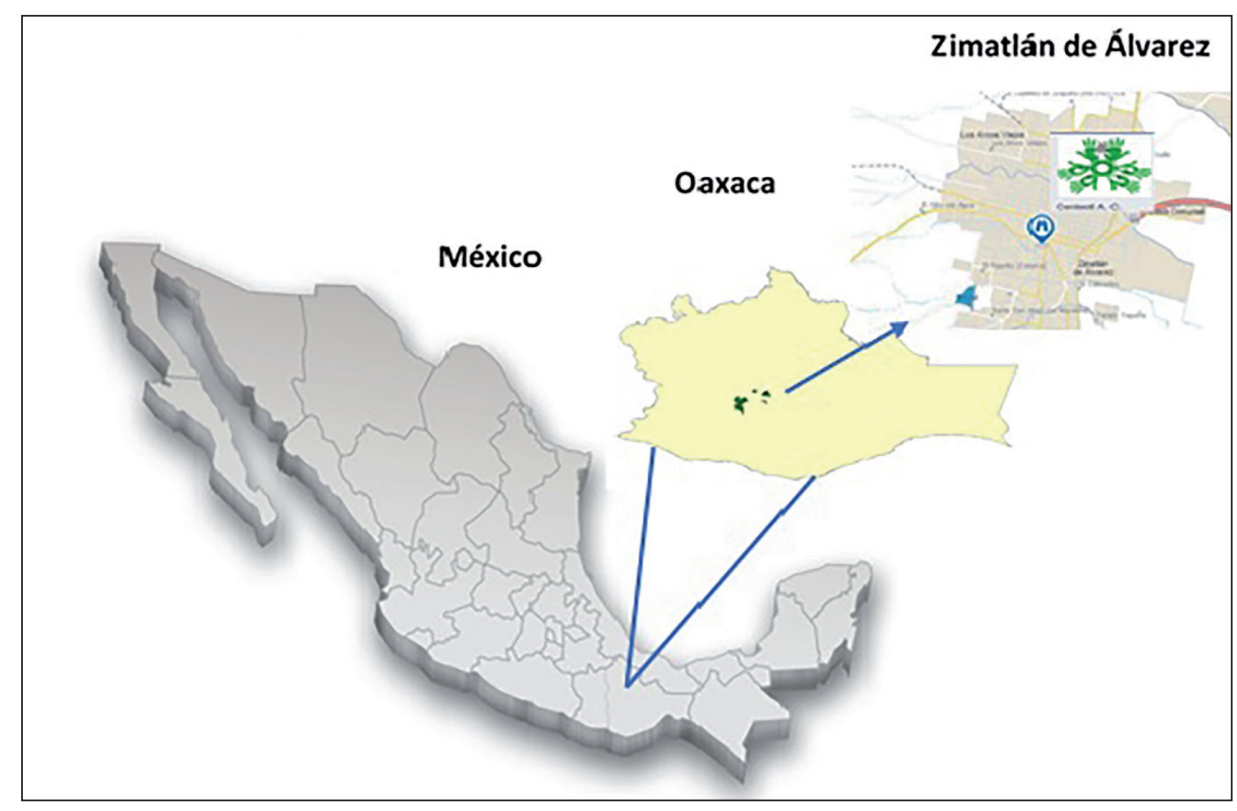

\subsection{Empresa social rural}

El estudio de Centéotl quedó integrada por dos dimensiones i) bienes y ii) servicios. i) La primera viene definida por dos indicadores: recurso otorgado, y capacitaciones y talleres. Según el indicador recurso otorgado el 67,6 por ciento recibió asesoría técnica y el 21.6 por ciento capacitaciones en el manejo del cultivo (Gráfica 1).

El 86,49 por ciento estuvo totalmente de acuerdo que las capacitaciones y talleres impartidos les ayudó a mejorar su producción (Gráfica 2).

La dimensión ii) de servicios, está integrada por dos indicadores: empresa Bancomunidad y programa Raíces y Horizontes.

En el indicador empresa Bancomunidad el 51,35 por ciento estuvo totalmente de acuerdo en que el recurso económico empleado para el cultivo de amaranto fue obtenido mediante préstamo (Gráfica 3).

En el indicador programa Raíces y Horizontes el 54,05 por ciento realiza una vez a la semana recolección de basura, reciclaje o alguna otra actividad relacionada con el cuidado del medio ambiente (Gráfica 4).

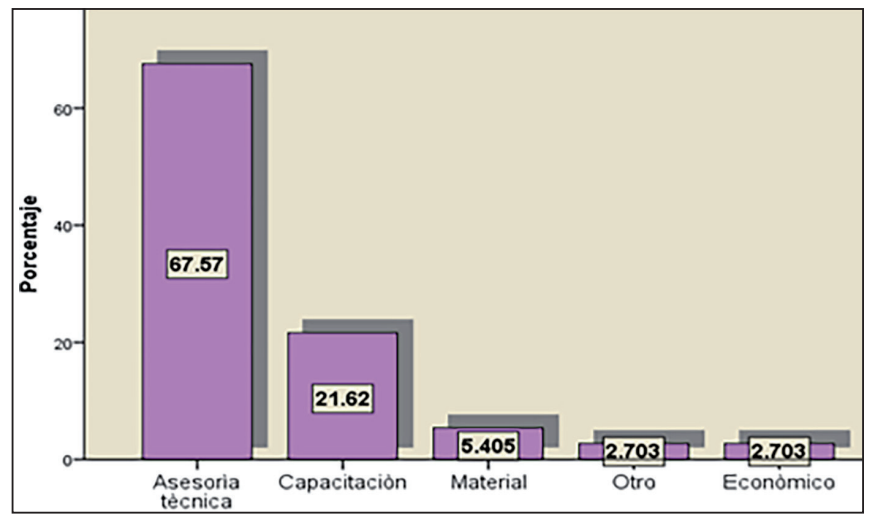

Gráfica 1. Apoyo otorgado por Centéotl. Fuente: Elaboración propia con el paquete estadístico IBM(SPSS) versión 25.

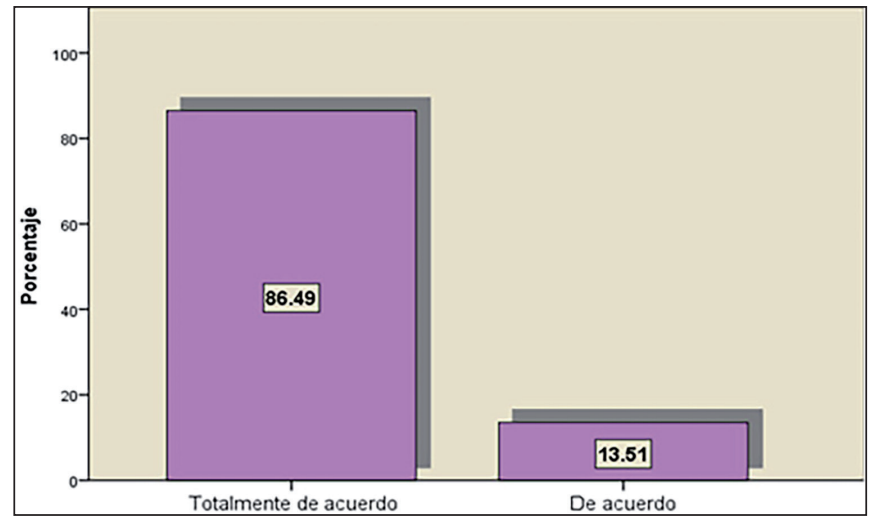

Gráfica 2. Capacitaciones y talleres otorgados por Centéotl. Fuente: Elaboración propia con el paquete estadístico IBM(SPSS) versión 25. 


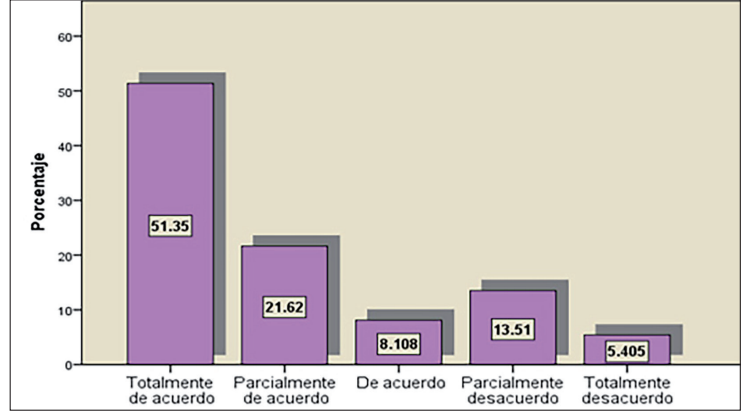

Gráfica 3. Recursos obetnidos de financiamiento. Fuente: Elaboración propia con el paquete estadístico IBM(SPSS) versión 25.

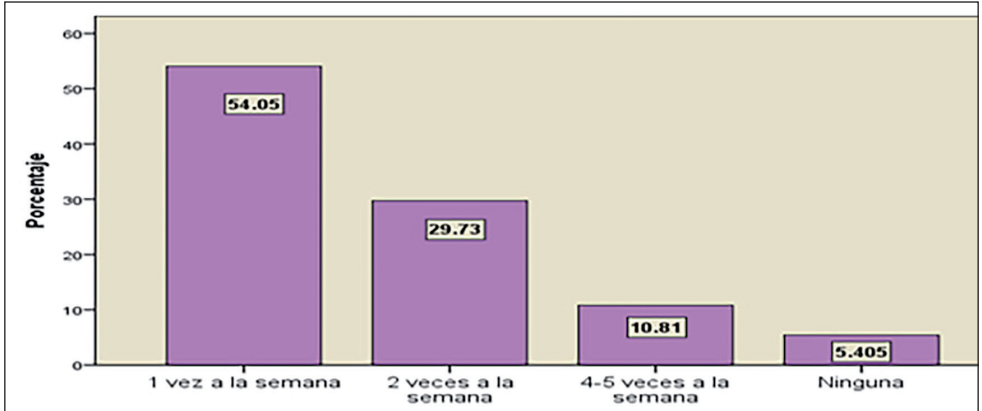

Gráfica 4. Promoción de actividades relacionadas al cuidado del medio ambiente. Fuente: Elaboración propia con el paquete estadístico IBM(SPSS) versión 25.

\subsection{Patrimonio agroalimentario}

El patrimonio agroalimentario quedó integrado por dos dimensiones i) técnicas de producción de amaranto y ii) conocimientos en el cultivo amaranto.

Las técnicas de producción están explicadas a través de tres indicadores: cultivo, recolección y consumo. Y éstas se centran en conocer el proceso de producción para establecer un pequeño huerto de amaranto.

En el indicador producción el 91,8 por ciento destina un terreno o parcela para la siembra de amaranto y el 62, 2 por ciento utiliza maquinaria agrícola para el cultivo (Gráficas 5 y 6).

En recolección el 83,8 por ciento dedica 6-8 horas diarias al cultivo de amaranto. Para la recolección de grano el 78,4 por ciento emplea también de 6-8 horas diarias (Gráficas 7 y 8).

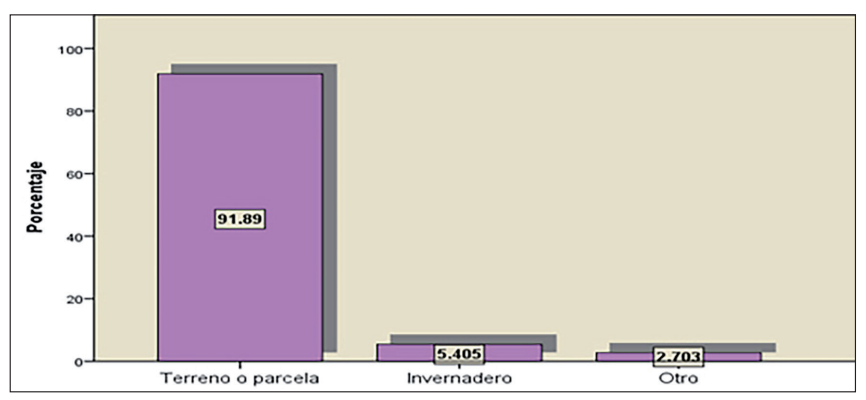

Gráfica 5. Área destinada al cultivo de amaranto.

Fuente: Elaboración propia con el paquete estadístico IBM(SPSS) versión 25.

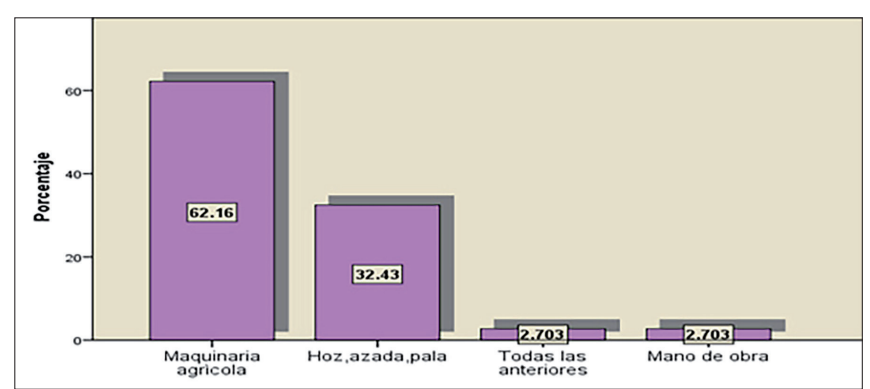

Gráfica 6. Herramientas y técnicas utilizadas en el cultivo de amaranto. Fuente: Elaboración propia con el paquete estadístico IBM(SPSS) versión 25.

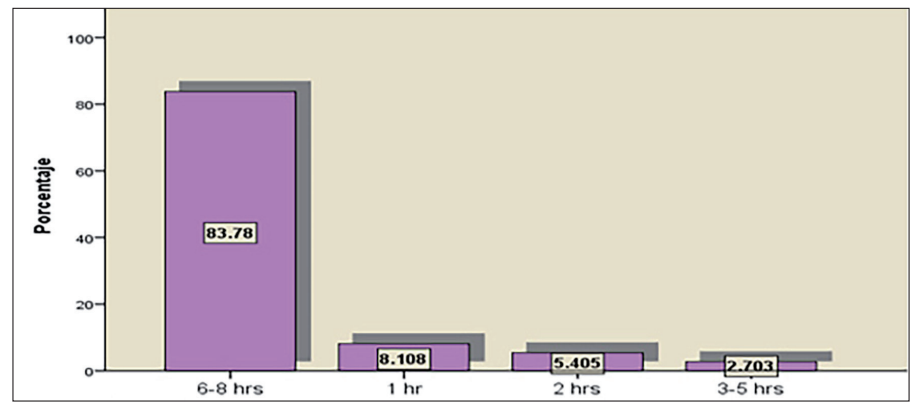

Gráfica 7. Tiempo destinado al cultivo de amaranto. Fuente: Elaboración propia con el paquete estadístico IBM(SPSS) versión 25.

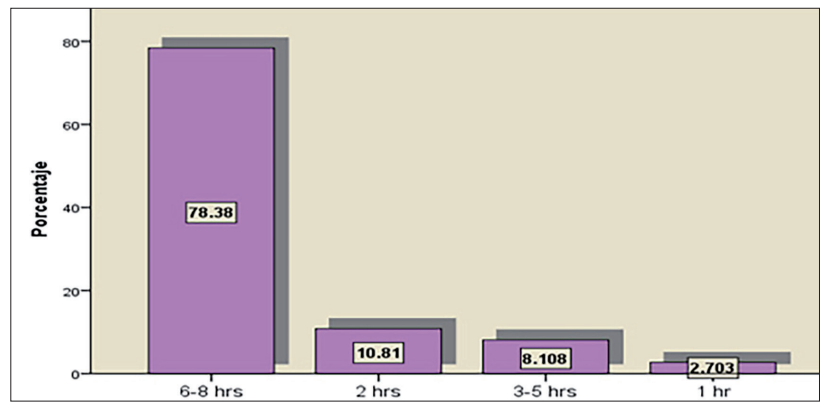

Gráfica 8. Tiempo destinado a la recolecta del grano de amaranto. Fuente: Elaboración propia con el paquete estadístico IBM(SPSS) versión 25. 
En cuanto al consumo, el 97,3 por ciento estuvo totalmente de acuerdo que el amaranto es una de las plantas con mayor calidad nutricional. El 81,1 por ciento recomienda el consumo de amaranto (semilla, harina, cereal y hojas). El 70,3 por ciento invita a consumir el amaranto de manera diaria (Gráficas 9, 10 y 11).

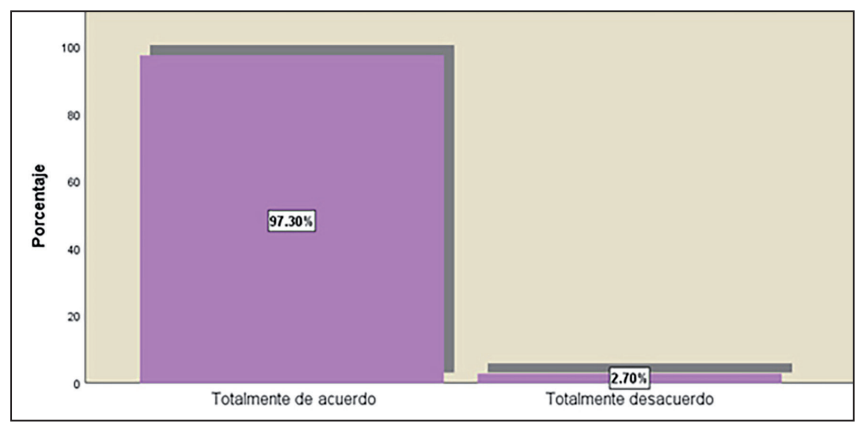

Gráfica 9. Amaranto planta con mayor calidad nutricional. Fuente: Elaboración propia con el paquete estadístico IBM(SPSS) versión 25.

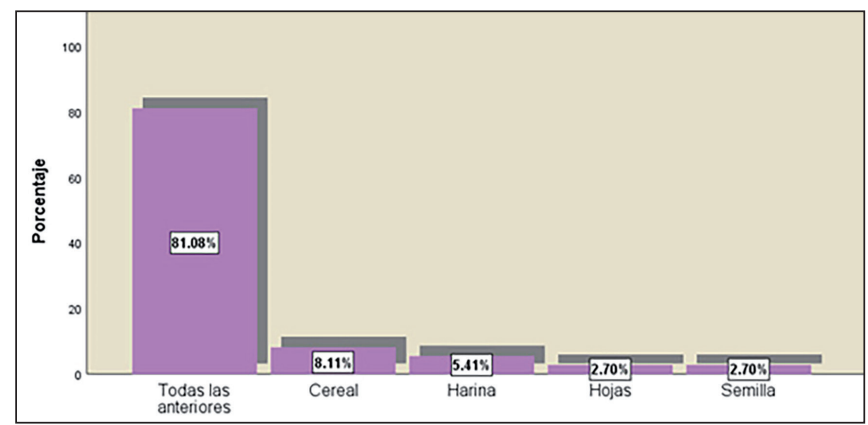

Gráfica10. Presentación para consumir amaranto. Fuente: Elaboración propia con el paquete estadístico IBM(SPSS) versión 25.

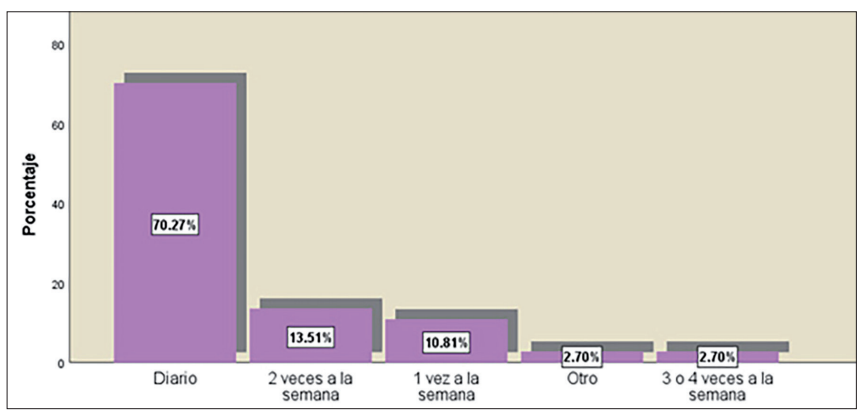

Gráfica11. Presentación para consumir amaranto. Fuente: Elaboración propia con el paquete estadístico IBM(SPSS) versión 25.

La dimensión ii) conocimientos en el cultivo de amaranto está integrada por dos indicadores (experiencia e interés en el cultivo de amaranto).

Los conocimientos en el cultivo de amaranto consideran la experiencia que tiene el productor de acuerdo a los años que practica ese cultivo, el interés en cultivarlo y el uso que le dan a ese alimento.

En el indicador experiencia en el cultivo, el 48,6\% han cultivado de 5 a 10 años amaranto, (Gráfica 12). En el indicador interés en el cultivo el 54,05\% cultiva el amaranto para consumo y venta (Gráfica 13).

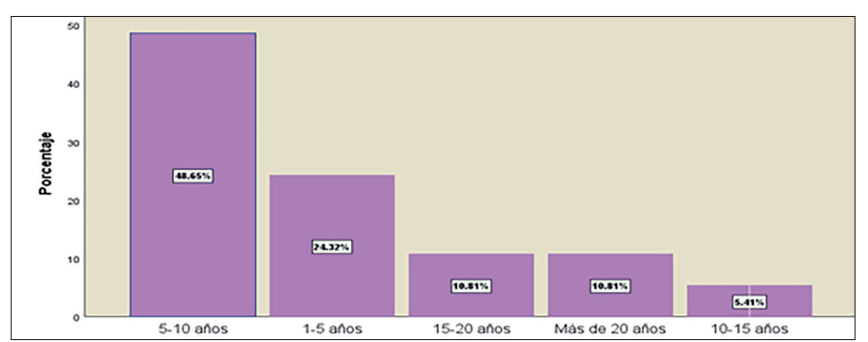

Gráfica 12. Años dedicados al cultivo de amaranto. Fuente: Elaboración propia con el paquete estadístico IBM(SPSS) versión 25.

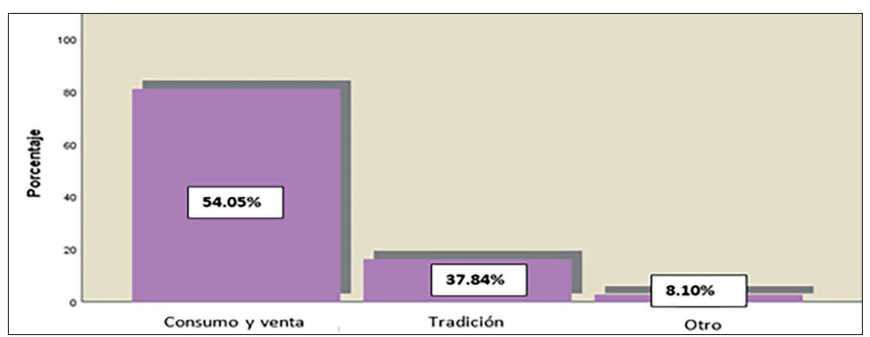

Gráfica 13. Interés en el cultivo de amaranto. Fuente: Elaboración propia con el paquete estadístico IBM(SPSS) versión 25 . 
Para comprobar la H1. La empresa social rural Centéotl realiza actividades que promueven la conservación del cultivo de amaranto como patrimonio agroalimentario; se analizaron los resultados considerando las respuestas que contribuían afirmativamente con más del 50 por ciento.

De esta forma, Centéotl oferta principalmente capacitaciones y talleres a productores y promueve la producción del cultivo de amaranto (81,08 por ciento). Seguido por los servicios de microcréditos que ofrece la empresa Bancomunidad (51,3 por ciento). En relación al patrimonio agroalimentario, resultaron relevantes las técnicas de producción del cultivo (91,4 por ciento), y el conocimiento de las propiedades nutricionales del amaranto, considerado con mayor calidad nutricional (97,3 por ciento) (Tabla 2).

Tabla 2. Resultados.

\begin{tabular}{|c|c|c|c|}
\hline & \multicolumn{3}{|c|}{ RESULTADOS } \\
\hline Variable & Dimensión & Indicador & Afirmación con más del 50\% \\
\hline \multirow{4}{*}{$\begin{array}{l}\text { Empresa social } \\
\text { rural (Centéotl) }\end{array}$} & \multirow[b]{2}{*}{ Bienes } & Recurso otorgado & $\begin{array}{l}67,6 \% \text { recibió asesoría técnica por parte de la } \\
\text { empresa social rural Centeótl A.C }\end{array}$ \\
\hline & & $\begin{array}{l}\text { Capacitaciones y } \\
\text { talleres }\end{array}$ & $\begin{array}{l}81,08 \% \text { estuvo totalmente de acuerdo que la } \\
\text { capacitación y taller proporcionado les apoya en } \\
\text { mejorar su producción. }\end{array}$ \\
\hline & \multirow{2}{*}{ Servicios } & $\begin{array}{l}\text { Empresa Bancomu- } \\
\text { nidad }\end{array}$ & $\begin{array}{l}51,3 \% \text { considera que el recurso económico para } \\
\text { el cultivo de amaranto es obtenido de financia- } \\
\text { miento. }\end{array}$ \\
\hline & & $\begin{array}{l}\text { Programa raíces y } \\
\text { horizontes }\end{array}$ & $\begin{array}{l}\text { Contribuyó en que el } 54,05 \% \text { realice activida- } \\
\text { des de recolección de basura y reciclaje en la } \\
\text { comunidad }\end{array}$ \\
\hline \multirow{9}{*}{$\begin{array}{l}\text { Patrimonio } \\
\text { agroalimentario }\end{array}$} & \multirow{7}{*}{ Técnicas de producción } & \multirow{2}{*}{ Producción } & $\begin{array}{l}91,4 \% \text { destina un terreno o parcela para la } \\
\text { siembra de amaranto }\end{array}$ \\
\hline & & & $\begin{array}{l}62,2 \% \text { utiliza maquinaria agrícola en espacios } \\
\text { que lo permita la condición del terreno }\end{array}$ \\
\hline & & \multirow{2}{*}{ Recolección } & $\begin{array}{l}83,78 \% \text { dedica } 6-8 \mathrm{~h} \text { diarias al cultivo de ama- } \\
\text { ranto }\end{array}$ \\
\hline & & & $\begin{array}{l}78,4 \% \text { dedica } 6-8 \text { h diarias para la recolección } \\
\text { de grano }\end{array}$ \\
\hline & & \multirow{3}{*}{ Consumo } & $\begin{array}{l}97,3 \% \text { considera que el amaranto es una de las } \\
\text { plantas con mayor calidad nutricional }\end{array}$ \\
\hline & & & $\begin{array}{l}81,8 \% \text { recomienda el consumo de amaranto en } \\
\text { semilla, harina, cereal y hojas }\end{array}$ \\
\hline & & & $\begin{array}{l}70,3 \% \text { invita a consumir el amaranto de manera } \\
\text { diaria }\end{array}$ \\
\hline & \multirow{2}{*}{$\begin{array}{l}\text { Conocimientos en el } \\
\text { cultivo de amaranto }\end{array}$} & $\begin{array}{l}\text { Experiencia en el } \\
\text { cultivo }\end{array}$ & $48,6 \%$ ha cultivado amaranto de 5-10 años \\
\hline & & Interés en el cultivo & $\begin{array}{l}54,05 \% \text { cultiva el amaranto para consumo y } \\
\text { venta }\end{array}$ \\
\hline
\end{tabular}

\section{DISCUSIÓN}

En relación al primer resultado, éste muestra que los bienes (recurso otorgado, capacitaciones y talleres) en el manejo y control del cultivo de amaranto, ofrecidos por parte de Centéotl a las familias productoras 
agrícolas, en estado de vulnerabilidad muchas de ellas, ha permitido adoptar el amaranto como cultivo complementario, y ha tenido doble efecto. Por un lado, cultivar y disponer de granos, hortalizas y legumbres con calidad nutricional, los cuales les permite asegurar la alimentación y nutrición de los miembros de la familia. Por otro lado, una vez satisfechas las necesidades de alimentación, los excedentes del cultivo los venden para obtener ingresos que utilizan para invertir en un nuevo ciclo de producción, tal como afirma Paredes y Sato (2018). En cuanto al efecto positivo de impartir recursos, capacitaciones y talleres se puede citar como ejemplo el caso de Colombia, donde por medio de la organización Agrosolidaria se trabaja con escuelas para revitalizar la agricultura familiar campesina, elaborar propuestas para mejorar condiciones de vida y contribuir en la revalorización en la agricultura (CAC, 2015).

Sin embargo, el menor impacto ha sido el servicio de microcrédito proporcionado por Bancomunidad, el cual es destinado para otros rubros, no en su totalidad para el cultivo de amaranto, pues solo el 51,3 por ciento de los productores utiliza financiamiento para sembrar. En ese sentido, Agrosolidaria trabaja desde otra perspectiva, esta se enfoca en finanzas comunitarias rurales, quien propone reestructurar los servicios de microfinanzas institucionales sobre la noción de reciprocidad, busca establecer vínculos solidarios entre asociados, en condición de copropietarios de su propio sistema financiero, generando organización financiera comunitaria a cambio de institución financiera prestadora de servicio. Dicho proceso se basa en construcción colectiva de conocimiento integrado por seminarios y talleres departamentales (CAC, 2015). Derivado de ello, se puede interpretar que es importante que Bancomunidad coordinado por Centéotl enfatice impartir talleres y seminarios con temas de organización y gestión de finanzas comunitarias (Martines, 2016; ONU, 2014), además de incluir temática de proyectos de inversión y producción de amaranto, pero de ser posible, con perspectiva de género, ya que cuando no se toma en consideración este aspecto, el microcrédito puede provocar mayor pobreza en las mujeres, al limitar sus posibilidades de participación política y social (García, 2014). Pero en general, los productores puedan aprovechar las ventajas de interés bajo que maneja ese servicio, mayor oportunidad de inversión y mayor oportunidad de producción y excedente, de tal manera que al igual que Agrosolidaria se genere una organización financiera comunitaria.

El segundo resultado, muestra que las familias han identificado en el cultivo de amaranto propiedades nutricionales que lo reconocen como una de las plantas con mayor calidad nutricional, y que el procedimiento para ser transformado en comida es fácil lo que les permite incluirlo en su dieta diaria, pues se ha comprobado que el amaranto tiene propiedades para ayudar a mantener la salud, y el valor nutritivo de sus granos implica que además de su contenido proteico, el espectro de aminoácidos y los niveles de vitaminas y minerales son excelentes (Mapes, 2015). De ahí, que al dar continuidad al cultivo se ha podido rescatar una planta ancestral con propiedades nutricionales, además de su permanencia de cultura a lo largo del tiempo y espacio, como medio de la transmisión de conocimientos entre generaciones sobre las bondades de ese cultivo y, preservar así el grano de amaranto como patrimonio agroalimentario (Agüero Teare, 2016; Echevarria et al., 2015; Inah, 2016).

\section{CONCLUSIONES}

Desde la economía solidaria, el estudio se realizó en la empresa social rural Centéotl y se observó que ésta, al impartir asesoría técnica, capacitaciones y talleres a familias productoras de amaranto, tiene la posibilidad de continuar con el cultivo, destinarlo para consumo y el excedente para el mercado. Esas ventajas permiten a las comunidades productoras que forman parte de Centéotl adoptar el cultivo de manera continua aprovechando su valor nutricional y rescatando su valor simbólico, de esta manera se reconoce su gran valor como patrimonio agroalimentario de la humanidad.

El estudio de caso sirvió para comprender las actividades y mecanismos que realizan los productores de amaranto para contrarrestar los problemas de seguridad alimentaria y por lo tanto conocer, los enormes esfuerzos que realizan las comunidades locales por conservar sus conocimientos tradicionales, formas y saberes en el cultivo de sus alimentos en una economía globalizada. A partir del análisis de las actividades que realiza la empresa social rural Centéotl se concluye que los bienes y servicios que ofrece a los produc- 
tores contribuyen en gran medida en el patrimonio agroalimentario. Sin embargo, se recomienda el diseño e implementación de una estrategia de capacitación integral cultural sobre experiencias y conocimientos tradicionales de otros productos agrícolas, que permitan preservar las técnicas de producción, recolección y consumo que se realizan en las comunidades, además la transmisión de esos conocimientos a las futuras generaciones, y con ello preservar cultivos que forman el patrimonio cultural inmaterial de muchos de los pueblos originarios en los países de América Latina, como ocurre con otros ejemplos (Agüero, 2016), en el caso del corredor Cusco-Puno, Perú, donde las prácticas agrícolas y culturales de la civilización incaica han sido conservadas y mejoradas involucrando sistemas de terrazas y cultivos en altas altitudes como la quinua, la maca y el maíz gigante; o como también sucede en el Archipiélago de Chiloé en Chile, para la producción de papas nativas y otros cultivos como la quinua, la frutilla y el ajo.

\section{REFERENCIAS}

Agüero Teare, T. (2016). Sitios SIPAM: rescate y valorización del patrimonio agrícola y cultural de un territorio. Oficina de Estudios y Políticas Agrarias, www.odepa.gob.cl. Recuperado de: https://www.odepa.gob.cl/wp-content/ uploads/2018/01/sipam2016.pdf, el 25 de enero de 2020.

Askunze, C. (2007). Economía solidaria. Educación para Askunze, C, 2007. Economia solidaria. En G. C. Muniain, Diccionario de Educación para el desarrollo (págs. 107-113). Bilbao: Hegoa.

Comité de seguridad alimentaria (2012). FAO Organización de las Naciones Unidas para la Alimentación y la Agricultura. Recuperado de: http://www.fao.org/3/a-mt648s.pdf, el 15 de mayo de 2019.

Confederación agrosolidaria Colombia, CAC (2015). Finanzas comunitarias. Recuperado de: http://www.agrosolidaria. org/index.php/ejes-de-trabajo/sistema-financiero, el 13 de mayo de 2019.

Echeverría, H., Etchegaray, M., Casanova, G., Barros, P. y Phillipi, D. (2015). Patrimonio agroalimentario a la conquista de los mercados. Revista Innova + agro, 1, 5-12.

De Jesús Contreras, D., Ramírez de la O, I., Viesca González, F. y Thome Ortiz, H. (2017). La búsqueda de la denominación de origen (do) del amaranto de Santiago Tulyehualco, Xochimilco, D.F. en Santiago Tulyehualco, Xochimilco. En U. A. México, Calificación, valorización y turismo. Aproximaciones al patrimonio agroalimentario (pp. 183-216). México: Creative Commons: Atribución-NoComercial-SinDerivar.

García Horta, J.L., Zapata Martelo, E., Valtierra Pacheco, E. y Garza Bueno, L. (2014). El microcrédito como estrategia para atenuar la pobreza de las mujeres, ¿cuál pobreza? Revista Estudios fronterizos, 15(30) Mexicali jul. /dic. 2014. Recuperado de http://www.scielo.org.mx/scielo.php?script=sci_arttext\&pid=S0187-69612014000200004 doi: https:// doi.org/10.21670/ref.2014.30.a04, el 20 de febrero de 2019.

Guber, R. (1991). El salvaje metropolitano. Buenos Aires. Legasa, 2001, La etnografía. Buenos Aires: Editorial Norma.

Hernández, S. R. (2010). Les métodos mixtos. En R. Hernández Sampieri, C. Fernández Collado y M. Baptista Lucio, Metodología de la Investigación (pp. 546). México: McGraw-Hill / Interamericana Editores, S.A. de C.V.

Herrera, T. F. (2012). Desarrollo rural en México políticas y perspectivas. Buenos Aires: MNEMOSYNE.

INAH (2016). Exposición muestra a la milpa como patrimonio biocultural. Recuperado de: http://www.inah.gob.mx/es/boletines/5600-exposicion-muestra-a-la-milpa-como-patrimonio-biocultural, el 14 de octubre de 2018.

Mapes Sánchez, E.C. (2015). El amaranto. Ciencia, Revista Academia Mexicana de Ciencias, julio-septiembre 2015, 66 (3), 8-15. Recuperado de : http://revistaciencia.amc.ed,.mx/images/revista/66_3/PDF/Amaranto.pdf, el 14 de febrero de 2020.

Martínez, S. (2016). Seguridad alimentaria, autosuficiencia y disponibilidad del amaranto en México. Revista Problemas de Desarrollo, 47, 107-132. Recuperado de: www.revistas.unam.mx/index.php/pde/article/download/55882/50171, el 13 de agosto de 2028. doi: https://doi.org/10.1016/j.rpd.2016.08.004

Martinez, M. (2017). Almanatura. Recuperado de: http://almanatura.com/2017/11/grupos-consumo-agroecologicos/, el 13 de agosto de 2018.

Márquez, D. J. (2002). Empleo y nuevas tareas rurales para el desarrollo local. Recuperado de: https: //dialnet.unirioja.es/, 57-69. doi: https://doi.org/10.14198/INGEO2002.29.06

Montecino, A. (2015). Patrimonio agroalimentario a la conquista de los mercados. Revista Innova + agro, 1, 51-52. 
Molano, R. (2018). Quinua y amaranto. Recuperado de: http://www.quinuayamaranto.com/nuestra-empresa, el 20 de agosto de 2018.

ONU (2014). Grupo de trabajo interinstitucional sobre economía social y solidaria. La economía social y solidaria y el reto del desarrollo sostenible. Ginebra. Recuperado de: http://unsse.org/wp-content/uploads/2014/08/Position-Paper_TFSSE_Esp1.pdf, el 28 de junio de 2018.

Ortiz, E. B. (2015). Empresas sociales hacia la sustentabilidad en México. Entretextos, 10.

Pallacán, C. (2016). Plataforma de territorios inteligentes. Recuperado de: http://www.fao.org/in-action/territorios-inteligentes/articulos/colaboraciones/detalle/es/c/455695/, el 14 de octubre de 2018.

Paredes, C y Sato, V. (2018). Eje temático: Ciencia Tecnología y Sociedad. Agricultura Autóctona para el desarrollo humano y social como única alternativa para la lucha contra el hambre en la región Salta - Argentina. Recuperado de: https:// www.unl.edu.ar/iberoextension/dvd/archivos/ponencias/mesa1/agricultura-autoctona-para-e.pdf, el 20 de abril de 2018.

Salcedo Aznal, A. y Marcillo Vaca, C. (2010). Economía solidaria. Ecuador: Fundación Iberoamericana para el Desarrollo, La Fundación Humanidad y Desarrollo.

Sanz, J. (2002). El sistema agroalimentario español: estrategias competitivas. Madrid: Mc Graw Hill.

Sanz, C. J. (2010). Los sistemas agroalimentarios locales: calidad, territorio y acción colectiva. Recuperado de: La Jornada. com.mx: https://www.jornada.com.mx/2010/02/13/sistemas.html

SEDESOL (2016). Impera la desnutrición y pobreza en Oaxaca. Recuperado de: https://www.vanguardia.com.mx/articulo/ impera-la-desnutricion-y-pobreza-en-oaxaca, el 24 de mayo de 2017.

Shamah, L. T., Amaya, C. M., y Cuevas, N. L. (2015). Desnutrición y obesidad: doble carga en México. Revista Rud revista digital universitaria unam.mx. Recuperado de: http://www.revista.unam.mx/vol.16/num5/art34/, el 22 de agosto de 2018.

Toledo, V. (2012). Red de etnoecología y patrimonio biocultural, 31-33. México Conacyt. Recuperado de: http: //etnoecologia.uv.mx/Red_paginaprincipal.html

Ubasart, G. (2009). Barcelona social. Dirección de acción social. Ayuntamiento de Barcelona. Recuperado de: http:// w110.bcn.cat/portal/site/ServeisSocials/menuitem.931633495bcd6167b4f7b4f7a2ef8a0c/indexe7b6.html?vgnextoid=594d505c8019d310VgnVCM1000001947900aRCRD\&vgnextchannel=594d505c8019d310VgnVCM1000001947900aRCRD\&lang=es_ES, el 15 de mayo de 2019.

UNESCO (2018). Patrimonio. Indicadores UNESCO de cultura para el desarrollo. Recuperado de: https://es.unesco.org/creativity/sites/creativity/files/digital-library/cdis/Patrimonio.pdf, el 23 de abril de 2018.

UNESCO (2017). Patrimonio cultural inmaterial. Actualizado el 26 de abril de 2019. Recuperado de: https://es.unesco. org/themes/patrimonio-cultural-inmaterial.

UNESCO (2015). Patrimonio cultural inmaterial y desarrollo sostenible. Recuperado de: https://ich.unesco.org/doc/src/34299-ES.pdf, el 22 de agosto de 2018.

Vasilanchis, D. G. (2006). Estrategias de investigación cualitativa. En Gialdino, I.V.: Estrategias de Investigación cualitativa (pp. 24-25). Barcelona, España: Gedisa, S.A.

Yin. R. (1994). Metodología. Recuperado de : http://catarina.udlap.mx/u_dl_a/tales/documentos/lmk/leal_m_a/capitulo4. pdf, el 22 de septiembre de 2018. 